\title{
Height-Preserving Transformations of Planar Graph Drawings
}

\author{
Therese Biedl* \\ David R. Cheriton School of Computer Science, University of Waterloo, \\ Waterloo, ON N2L 3G1, Canada \\ biedl@uwaterloo.ca
}

\begin{abstract}
There are numerous styles of planar graph drawings, such as straight-line drawings, poly-line drawings, orthogonal graph drawings and visibility representations. Given a planar drawing in one of these styles, can it be converted it to another style while keeping the height unchanged? This paper answers this question for (nearly) all pairs of these styles, as well as for related styles that additionally restrict edges to be $y$-monotone and/or vertices to be horizontal line segments. These transformations can be used to develop new graph drawing results, especially for height-optimal drawings.
\end{abstract}

Keywords: Planar graph drawing, poly-line drawing, straight-line drawing, orthogonal drawing, visibility representation, minimizing height.

\section{Introduction}

Let $G=(V, E)$ be a simple graph with $n=|V|$ vertices and $m=|E|$ edges. All graphs in this paper are planar, i.e., can be drawn without crossings. Some of the most commonly used drawings styles are the following: (1) A straightline drawing is a drawing where vertices are points and edges are straight-line segments between their endpoints. Any planar graph has a planar straight-line drawing in an $O(n) \times O(n)$-grid [8] [17]. (2) A poly-line drawing (called mixed model in [12]) is a drawing where vertices are points and edges are polygonal curves. Some results exist for poly-line drawings for which no equivalent straightline drawing result is known; for example Kant gave drawings in an $O(n) \times O(n)$ grid with large minimum angle [12. (3) A (2-directional) visibility representation is a drawing where vertices are axis-aligned boxes and edges are horizontal or vertical line segments. Every planar graph has a visibility representation in an $O(n) \times O(n)$-grid [16] 20] 21]. (4) An orthogonal (box-)drawing is a drawing where all vertices are axis-aligned boxes and edges are polygonals curves for which all line segments are horizontal or vertical. Every planar graph has an orthogonal drawing in an $O(n) \times O(n)$-grid [18].

\footnotetext{
* Supported by NSERC and the Ross and Muriel Cheriton Fellowship. The author would like to thank the referees of a preliminary version for helpful comments, and Fabrizio Frati and Géza Tóth for pointing out reference [15].
}

C. Duncan and A. Symvonis (Eds.): GD 2014, LNCS 8871, pp. 380-391 2014.

(C) Springer-Verlag Berlin Heidelberg 2014 
The research of this paper was driven by the following question: Does one really need to develop algorithms and lower bounds for these four drawing styles separately? Or is it possible to take a drawing in one style, and convert it directly to another style, preserving some of the features along the way? This would significantly simplify the development of algorithms and lower bounds.

This paper studies the existence of such transformations under the objective of maintaining the height of the drawing. It also includes some discussion on the width, and shows that transformations that maintain the height sometimes require an exponential blow-up in the width. Due to some prior work and applications, two variations of the drawing styles are included. First, a drawing is called $y$-monotone if each edge is drawn as a $y$-monotone curve. Every straightline drawing and every visibility representation is $y$-monotone, but orthogonal drawings and poly-line drawings need not be. Second, a drawing is called flat if every vertex is represented by a horizontal line segment. Every straight-line drawing and every poly-line drawing is automatically flat, but orthogonal drawings and visibility representations need not be. Fig. 1]lists the results, which can be summarized as follows:

- Straight-line drawings, $y$-monotone poly-line drawings, flat visibility representations and flat $y$-monotone orthogonal drawings are equivalent, where "equivalent" means "can be transformed into each other such that the height of the drawings remains the same".

- Poly-line drawings and orthogonal drawings are also equivalent.

- $y$-monotone orthogonal drawings are strictly between orthogonal drawings and visibility representations.

- Visibility representations are between orthogonal $y$-monotone drawings and straight-line drawings, where the latter relationship may be an equality.

The transformations keep the width linearly bounded or better, with the notable exception of creating straight-line drawings: here we show that an exponential increase in width is required for some graphs if the height must stay the same.

These results have some applications given in Section 6. Most importantly, they allow to derive some height-bounds for which no direct proof appears known, and they can be used to formulate some NP-hard graph drawing problems as integer programs.

\section{Preliminaries}

Throughout this paper $G$ denotes a planar graph, and $\Gamma$ denotes a planar drawing of $G$ that represents vertices of $G$ as axis-aligned boxes (possibly degenerated into horizontal segments or points) and edges of $G$ as polygonal curves (possibly straight line segments). The common end of two line segments in a polygonal curve is called a bend. $\Gamma$ is called $y$-monotone if for all edges the $y$-coordinates monotonically increase while going from one end to the other; horizontal segments are allowed. $\Gamma$ is called flat if all vertices are horizontal segments.

Call a drawing a grid-drawing if all corners of vertex-boxes and all endpoints of segments of polygonal curves have integer coordinates. A grid drawing is said 


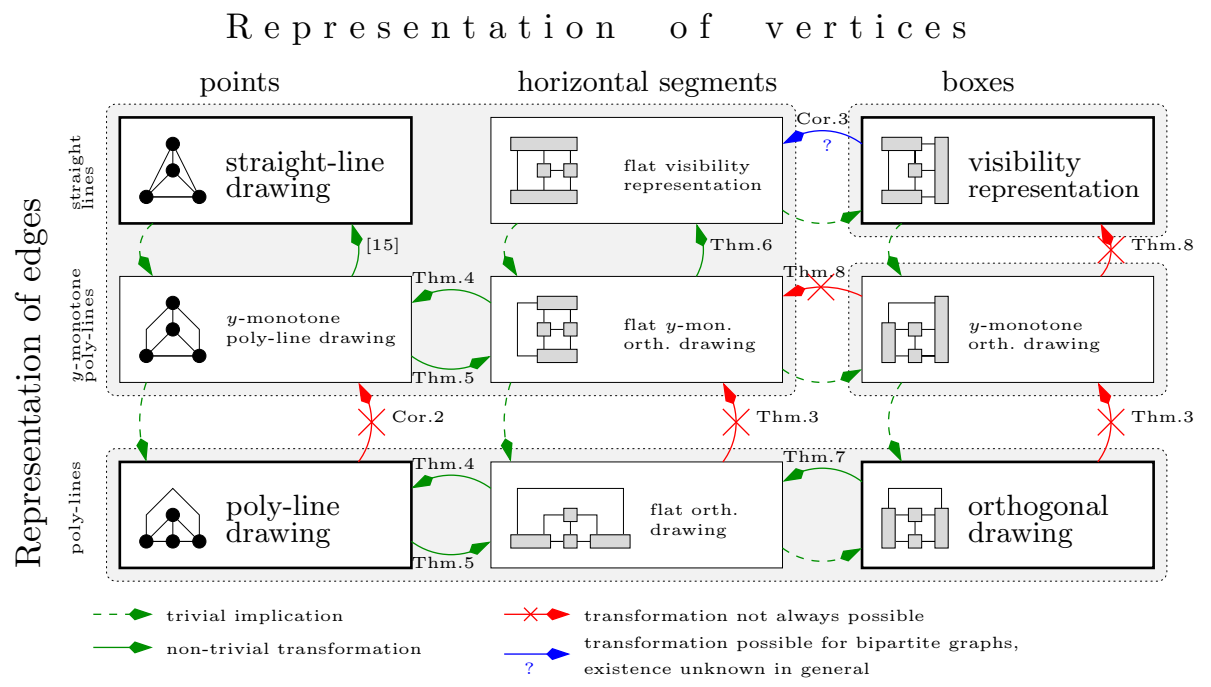

Fig. 1. Summary of height-preserving transformations

to have width $w$ and height $h$ if (possibly after translation) vertices and bends are placed on the $[1, w] \times[1, h]$-grid. The height is thus measured by the number of rows, i.e., horizontal lines with integer $y$-coordinates that intersect the drawing 1 Drawings in this paper are required to be grid-drawings, with some exceptions for ease of description that will be pointed out as they occur.

The goal is to transform a planar drawing $\Gamma$ into a different planar drawing $\Gamma^{\prime}$ which has the same height but uses a different drawing style. If $\Gamma$ and $\Gamma^{\prime}$ are both flat, then these transformations will have two useful properties as follows. $\Gamma^{\prime}$ preserves $y$-coordinates if any vertex has the same $y$-coordinate in $\Gamma$ and $\Gamma^{\prime}$. This is well-defined since both drawings are flat. $\Gamma^{\prime}$ preserves left-to-rightorders if, scanning each row from left to right, one encounters the same edges and vertices, in the same order, in both $\Gamma$ and $\Gamma^{\prime}$. One can easily show that if $\Gamma$ is planar and $\Gamma^{\prime}$ preserves $y$-coordinates and left-to-right-orders, then $\Gamma^{\prime}$ is also planar and has the same height. Furthermore, $\Gamma^{\prime}$ is $y$-monotone if $\Gamma$ was.

\section{Point-Drawings}

This section considers transformations among drawing styles that represent vertices by points. One of the few existing results about height-preserving transformations is by Pach and Tóth:

Theorem 1. [15] Any planar y-monotone poly-line drawing $\Gamma$ can be transformed into a planar straight-line drawing $\Gamma^{\prime}$ with the same height.

${ }^{1}$ In the pictures vertex representations are thickened for ease of readability, but this is not counted towards the height; e.g. the drawings in Fig. 1 have height 3. 


\subsection{Exponential Width}

Pach and Tóth did not analyze what happens to the width during the transformation. In fact, they did not even worry about achieving integer coordinates, but it is clear that this can be done with minor modifications. One can show that the width must increase exponentially for some graphs:
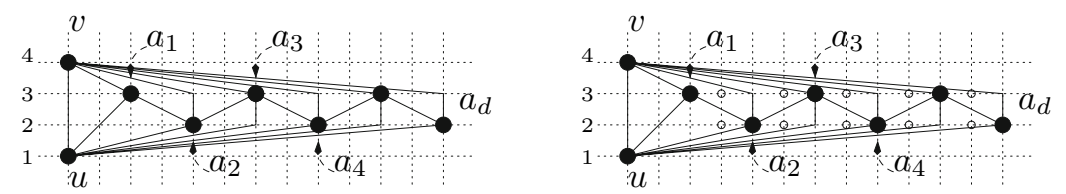

Fig. 2. (Left) A planar graph. (Right) Inserting vertices into inner faces.

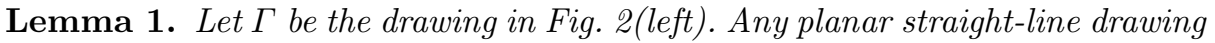
$\Gamma^{\prime}$ that preserves the $y$-coordinates and left-to-right-orders of $\Gamma$ has width at least $\frac{1}{3} 2^{d+1}$.

Proof. Denote by $x(w)$ the $x$-coordinate of vertex $w$ in $\Gamma^{\prime}$. Assume that $x(v) \leq$ $x(u)$; the other case is proved similar and in fact gives an even larger width bound. For ease of arithmetic operations, assume the drawing has been translated so that $x(v)=0$. One can now show by induction on $i$ (details are omitted) that

$$
x\left(a_{2 i-1}\right) \geq \frac{1}{3}\left(x(u)+2^{2 i}\right)-1 \quad \text { and } \quad x\left(a_{2 i}\right) \geq \frac{1}{3}\left(2 x(u)+2^{2 i+1}\right)-1
$$

for $i \geq 1$. This implies the result.

Theorem 2. There exists a graph $H$ that has a planar straight-line drawing of height 4 , but any such drawing has width at least $\frac{1}{3} 2^{n / 3}$.

Proof. The graph $H$ is obtained by taking the graph $G$ from Fig. 2 (left) with $d \geq 11$ and inserting into each inner face except $\left\{u, v, a_{1}\right\}$ a new vertex adjacent to the three vertices of the face. Note that $H$ is triangulated and has $3 d$ vertices. It has a $y$-monotone poly-line drawing on four rows (see Fig. 2(right)), and hence by Thm. 1 also a straight-line drawing on 4 rows.

Let $\Gamma_{H}$ be an arbitrary planar straight-line drawing of $H$ that uses four rows. Let $\Gamma_{G}$ be the induced planar straight-line drawing of $G$. One can argue (details are omitted) that $\Gamma_{G}-a_{d}$ preserves, after possible horizontal and vertical flips, the $y$-coordinates and left-to-right-orders of the drawing of Fig. 2(left). Hence it requires width at least $\frac{1}{3} 2^{d}$ by the previous lemma and $\Gamma_{H}$ cannot be smaller.

One can easily show that $H$ requires at least 4 rows in any straight-line drawing. Thm. 2 hence has the following consequence:

Corollary 1. There exists an infinite class of planar graphs such that any planar straight-line drawing that has optimal height has exponential area. 


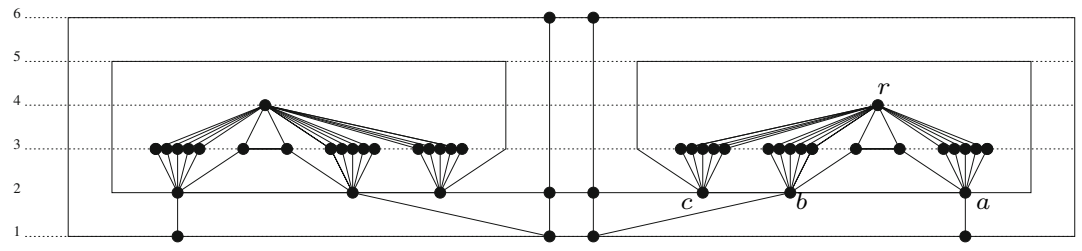

Fig. 3. A graph that can be drawn on 6 rows, but not if edges must be $y$-monotone

\section{$3.2 y$-monotonicity}

Thm. 1 used $y$-monotonicity of the poly-line drawing. One can show that $y$ monotonicity is required.

Theorem 3. The graph in Fig. [3 has a planar poly-line drawing on 6 rows, but no planar y-monotone orthogonal drawing on 6 rows.

Proof. (Sketch) Due to the $K_{2,5}$ 's between $r$ and each of $\{a, b, c\}$, vertices $a, b, c$ must all be in the same row. This makes it impossible to draw cycle $a-b-c-a$ with $y$-monotone curves.

Corollary 2. There exists a planar graph with a planar poly-line drawing on 6 rows that has no planar straight-line drawing on 6 rows.

Proof. If the graph in Fig. 3 had a straight-line drawing on 6 rows, then by Thm. 5 (below) it would have a $y$-monotone orthogonal drawing on 6 rows, contradicting Thm. 3.

\section{$4 \quad$ Flat Drawings}

This section is devoted to drawings where vertices are horizontal segments or points, and in particular aims to show that poly-line drawings are equivalent to flat orthogonal drawings as far as height is concerned. Note that neither of these implications is trivial: A poly-line drawing allows slanted line segments while a flat orthogonal drawing does not, and a flat orthogonal drawing allows horizontal segments for vertices while a poly-line drawing does not.

Theorem 4. Any planar flat orthogonal drawing $\Gamma$ can be transformed into a planar poly-line drawing $\Gamma^{\prime}$ of the same height that preserves $y$-coordinates and left-to-right orders. $\Gamma^{\prime}$ has no more width than $\Gamma$. Moreover, if $\Gamma$ is $y$-monotone then so is $\Gamma^{\prime}$.

Proof. First insert pseudo-vertices obtained by subdividing edges at bends and whenever a vertical segment of an edge crosses a row. Any pseudo-vertex is located on a grid-point since the edge segment was vertical, so this does not 
increase the width. Now within each row $r$ enumerate the vertices and pseudovertices as $w_{1}, \ldots, w_{k}$ from left to right, and assign $w_{i}$ to the point $(r, i)$; clearly this preserves $y$-coordinates and left-to-right-orders. Since $w_{1}, \ldots, w_{k}$ had integer $x$-coordinates before, the $x$-coordinate of each can only decrease. Due to the pseudo-vertices edge-segments connect vertices in the same or two adjacent rows, so they can be drawn without crossings. Replacing the pseudo-vertices by bends gives the desired poly-line drawing $\Gamma^{\prime}$. The claim on height and $y$-monotonicity holds since $y$-coordinates and left-to-right-orders are preserved.

Theorem 5. Any planar poly-line drawing $\Gamma$ can be transformed into a planar flat orthogonal drawing $\Gamma^{\prime}$ of the same height that preserves $y$-coordinates and left-to-right orders. If $\Gamma$ is $y$-monotone, then so is $\Gamma^{\prime}$.

Proof. Insert pseudo-vertices at bends and whenever a segment of an edge crosses a row, temporarily allowing non-integer $x$-coordinates. Now within each row $r$ enumerate the vertices and pseudo-vertices as $w_{1}, \ldots, w_{k}$ from left to right. In $\Gamma^{\prime}$, replace each $w_{i}$ by a box of width $\max \left\{1, \operatorname{deg}^{u p}\left(w_{i}\right), \operatorname{deg}_{\text {down }}\left(w_{i}\right)\right\}$, where $\operatorname{deg}^{u p}\left(w_{i}\right)$ and $\operatorname{deg}_{\text {down }}\left(w_{i}\right)$ are the number of neighbours above and below $w_{i}$, respectively. Place these boxes in row $r$ in the same left-to-right order.

If an edge was drawn horizontally in $\Gamma$, then draw it horizontally in $\Gamma^{\prime}$ as well. Each non-horizontal edge ends in two adjacent rows due to the pseudo-vertices. Connect the edges between two adjacent rows using VLSI channel routing (see e.g. [13]), using two bends per edge and lots of new rows with non-integer $y$ coordinates that contain horizontal edge segments and nothing else.

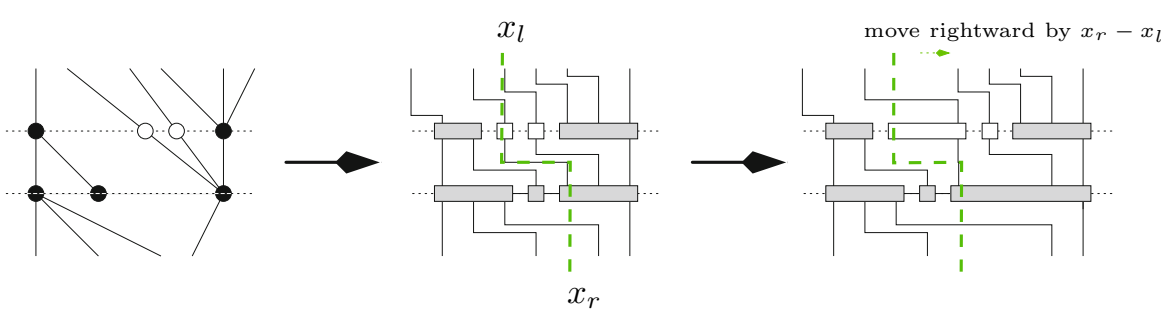

Fig. 4. Replace bends and row-crossings by pseudo-vertices (white). Replace points by boxes and route non-horizontal segments as zig-zags. Then remove zig-zags by shifting parts of the drawing rightward.

Each non-horizontal edge is now routed as a "zig-zag", ending vertically at its endpoints. It is well known (see e.g. [3]) that such a zig-zag can be removed as follows: Let $\left[x_{l}, x_{r}\right] \times y_{s}$ by the horizontal segment of a zig-zag. Let $M$ be all bends and endpoints of vertices with $x$-coordinate exceeding $x_{r}$, or $y$-coordinate at least $y_{s}$ and $x$-coordinate at least $x_{l}$. Then move all points in $M$ rightwards, i.e., add $x_{r}-x_{l}$ to their $x$-coordinate. See also Fig. 4. Notice that this preserves $y$-coordinates and planarity, and eliminates the horizontal segment. 
Repeating this for all zig-zags removes all horizontal segments with noninteger coordinates and hence gives the desired height and a flat visibility representation of the graph with pseudo-vertices. Any pseudo-vertex can now be removed and replaced by a bend or line-segment, if needed, to give an orthogonal drawing that satisfies all conditions.

A very similar construction converts $y$-monotone flat orthogonal drawings into flat visibility representations.

Theorem 6. Any planar flat $y$-monotone orthogonal drawing $\Gamma$ can be transformed into a planar flat visibility representation $\Gamma^{\prime}$ of the same height that preserves $y$-coordinates and left-to-right orders.

Proof. Assume that there exists an edge $e$ in $\Gamma$ that attaches horizontally at one endpoint $v$ and has bends. Since $\Gamma$ is flat, $v$ is a horizontal segment, which can expand until it covers the bend of $e$ nearest to $v$. Afterwards $e$ attaches vertically at $v$. Repeat this until all edges with bends attach vertically at both endpoints.

If there are now no bends then the claim holds. Otherwise, let $e$ be an edge with bends; by the above it attaches vertically at both its endpoints. Since $e$ is drawn $y$-monotone, it attaches at the top of one endpoint and the bottom of the other endpoint, and inbetween must have a zig-zag. As before (see Fig. 4) such a zig-zag can be removed by shifting parts of the drawing rightwards. This does not add height or bends. Applying this to all edges that have bends gives a planar visibility representation.

One height-transformation among point drawings and flat drawings remains to be studied: Can flat orthogonal drawings be made $y$-monotone without increasing the height? The answer here is "no" since the graph of Thm. 3 has a poly-line drawing on 6 rows, hence also (by Thm. 5) a flat orthogonal drawing on 6 rows, but it has no $y$-monotone orthogonal drawing, much less one that is flat.

Summary: Combining all the above results yields that straight-line drawings, $y$-monotone poly-line drawings, $y$-monotone flat orthogonal drawings and flat visibility representations are equivalent with respect to the height of planar drawings. 2 Poly-line drawings and flat orthogonal drawings are also equivalent, and the former group sometimes requires strictly more height than the latter.

Width Considerations: For Thm. 1 the width may have to increase exponentially (Thm. 3). For Thm. 4 the width did not increase. For Thm. 5 and 6 the width may increase, but after eliminating all zig-zags many columns are redundant: They contain no vertical edge, nor are they the only column of a vertex. In an orthogonal drawing redundant columns can simply be deleted. One can easily show the following:

${ }^{2}$ Historical note: At WAOA'12 [2], I claimed that any flat visibility representation can be transformed into a straight-line drawing of the same height directly, without using [15. Unfortunately the transformation in 2] is incorrect since the resulting drawing may not be planar; details are in the full version. 
Lemma 2. Any visibility representation of a connected graph has width at most $\max \{m, n\}$ after deleting redundant columns.

Hence for Thm. 6 the width is $\max \{m, n\}$. For Thm. 5 it is at $\operatorname{most} \max \{n, m\}+$ $b$, where $b$ is the number of local minima and maxima of polygonal curves in the poly-line drawing, since all other pseudo-vertices are eliminated when removing zig-zags.

\section{Box-Drawings Made Flat}

Theorem 7. Any planar orthogonal drawing $\Gamma$ can be transformed into a planar flat orthogonal drawing $\Gamma^{\prime}$ of the same height.

Proof. For any vertex $v$, let $r$ be a row that intersects the box $B(v)$ representing $v$ and set $B^{\prime}(v):=B(v) \cap r$. Any edge that attached vertically at $B(v)$ can be extended to end at $B^{\prime}(v)$ instead. Any edge that attached at $B(v)$ horizontally in row $r$ also attaches at $B^{\prime}(v)$. Any edge $e$ that attached at $B(v)$ horizontally, but not in row $r$, is re-routed by inserting a bend where $e$ attached at $B(v)$, and then going vertically towards $B^{\prime}(v)$. Now vertical edge segments at $v$ may overlap, but this can easily be remedied by replacing the leftmost and rightmost column of $B(v)$ by sufficiently many columns. See Fig. 5 ,

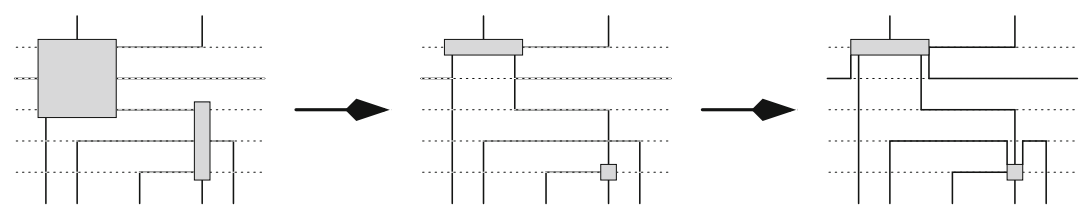

Fig. 5. Transforming orthogonal drawings into flat orthogonal drawings

Thm. [7 does not generally preserve $y$-monotonicity, and this is unavoidable.

Theorem 8. The planar graph in Fig. 6 has a y-monotone orthogonal drawing on 6 rows, but it has no planar y-monotone flat orthogonal drawing on 6 rows, and also no planar visibility representation on 6 rows.

Proof. (Sketch) The $4 \times 2$-grids force vertices $v, w$ onto row 5 and $v^{\prime}, w^{\prime}$ onto row 2 . The two cycles $\{v, w, c\}$ and $\left\{v^{\prime}, w^{\prime}, c^{\prime}\right\}$ enclose $x$ and $x^{\prime}$, respectively, which forces $c$ to span both rows 3 and 4 .

One transformation remains to be studied: Can every visibility representation be turned into a flat visibility representation? A variation of Thm. 7 shows that this is possible for bipartite graphs.

Corollary 3. Any planar visibility representation of a bipartite graph $G$ can be transformed into a planar flat visibility representation of the same height. 


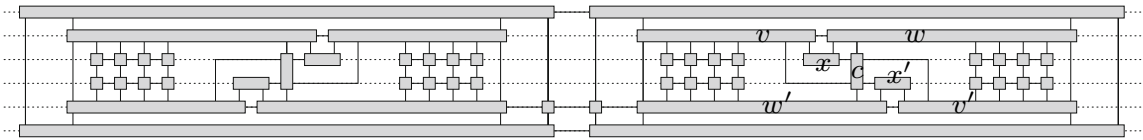

Fig. 6. A graph that has an orthogonal $y$-monotone on 6 rows, but not if vertices must be horizontal segments or edges have no bends

Proof. Since $G$ is bipartite, it has a vertex-coloring with 2 colors. Proceed as in Thm. 7, but use the topmost row intersecting $B(v)$ for each vertex $v$ in one color-class, and the bottommost row for each vertex in the other color-class. Each vertical edge remains vertical. Each horizontal edge becomes $y$-monotone since it connects two differently-colored vertices. So the result is a flat $y$-monotone orthogonal drawing, which can be converted into a flat visibility representation by Thm. 6,

It remains open whether visibility representations of arbitrary graphs can also be made flat without increasing the height.

\section{Applications}

This section highlights some applications of the above results.

Best Drawing Styles for Lower Bounds and Algorithms: Whenever possible, lower bounds for the height of planar graph drawings should be done for poly-line drawings or for orthogonal drawings: by the above transformations such a lower bound then also holds for visibility representations and straight-line drawings. Vice versa, algorithms to create planar graph drawings of small height should ideally be for straight-line drawings; they then also hold for all other models. Alternative, algorithms could be given for flat visibility representations; the same height-bound then also holds for straight-line drawings and all other models, though the width does not transfer.

Drawings of Small Height: Two examples of using height-transformations to achieve new results shall suffice:

Theorem 9. Every outer-planar graph $G$ has a planar straight-line drawing of height $O(p w(G))$, which is in $O(\log n)$. Here $p w(G)$ is the so-called pathwidth of the graph 3

Proof. Every outer-planar graph can be made 2-connected while increasing the pathwidth by at most a constant factor [1. Every 2-connected outer-planar graph has a flat visibility representation of height $O(p w(G))$ 2. This flat visibility representation is a flat $y$-monotone orthogonal drawing, which by Thms. 4 and 1 can be turned into a straight-line drawing of the same height.

${ }^{3}$ A similar result was claimed in [2], but required the (incorrect) transformation in that paper. 
Theorem 10. Any 4-connected planar graph has a planar flat visibility representation of height at most $\lfloor n / 2\rfloor$.

Proof. Any 4-connected planar graph has a straight-line drawing where the sum of the width and height is at most $n$ [14. After possible rotation, the height is at most $\lfloor n / 2\rfloor$, and with Thms. 5 and 6 one obtains a flat visibility representation of height at most $\lfloor n / 2\rfloor$.

The best previous bound on the height of visibility representations of 4connected planar graphs was $\left\lceil\frac{3 n}{4}\right\rceil[10]$.

Simplified Proofs: Some results are known about graphs that have planar straight-line drawings of height $h$. In particular, any such graph has pathwidth at most $h$ [7, and there exists an algorithm that is fixed-parameter tractable in $h$ to test whether a graph has a straight-line drawing of height at most $h[6]$. By the results in this paper a graph has a straight-line drawing of height $h$ if and only if it has a flat visibility representation of height $h$. While "simplicity of proof" is a subjective matter, in my opinion the presentation of both of the above results can be simplified if one shows the properties for flat visibility representations of height $h$, rather than straight-line drawings.

HH-drawings: In a previous paper [19] we studied HH-drawings, where a planar graph $G$ with a vertex partition $V=A \cup B$ should be drawn such that all vertices in $A$ have positive $y$-coordinates and all vertices in $B$ have negative $y$ coordinates. We gave a condition that is necessary for straight-line HH-drawings and sufficient for $y$-monotone poly-line HH-drawings. By the result by Pach and Tóth (Thm. 1) the condition is hence necessary and sufficient for straight-line HH-drawings. This proves:

Theorem 11. Any planar bipartite graph has a planar straight-line HH-drawing. Testing whether a planar graph with a given partition has a planar straight-line $H H$-drawing can be done in linear time.

Minimizing Heights Using Integer Programs: In a recent paper, we developed integer program (IP) formulations for many graph drawing problems where vertices and edges are represented by axis-aligned boxes [4. In particular, we gave an integer program with $O\left(h n^{2}\right)$ variables and constraints to test whether a graph has a bar-visibility representation (i.e., a visibility representation where vertices are horizontal segments and all edges are vertical) that has height $h$. It is not hard to modify this IP so that horizontal edges are allowed as well; then it tests the existence of flat visibility representations of height $h$. Since straight-line drawings and flat visibility representations are equivalent with respect to height, therefore:

Theorem 12. There exists an integer program with $O\left(h n^{2}\right)$ variables and constraints to test whether a graph has a planar straight-line drawing of height $h$.

While an algorithm was already known to test whether $G$ has a planar drawing of height at most $h\left[6\right.$, its rather large run-time of $O\left(2^{32 h^{3}}\right.$ poly $\left.(n)\right)$ means that solving the above integer program might well be faster in practice. 
Upward Drawings: A directed acyclic graph has an upward planar drawing if it has a planar straight-line drawing such that for any directed edge $v \rightarrow w$ the $y$-coordinate of $v$ is smaller than the $y$-coordinate of $w$. Testing whether a graph has an upward planar drawing is NP-hard 9]. There exists a way to formulate " $G$ has an upward planar drawing" as either IP or as a Satisfiability-problem, using partial orders on the edges and vertices [5]. The transformations in this paper give a different way of testing this via IP:

Lemma 3. A directed acyclic graph has an upward planar drawing if and only if it has a planar bar-visibility representation where for all edges the head is above the tail.

Proof. Any straight-line upward planar drawing can be transformed into a flat visibility representation using Thm. 5]and 6. Since $y$-coordinates are unchanged, any edge is necessarily drawn vertical with the head above the tail, so this is a bar-visibility representation. Vice versa, given such a bar-visibility representation, it can be transformed into a $y$-monotone poly-line drawing (Thm. 4) and from there into a straight-line drawing (Thm. 1). Since $y$-coordinates and leftto-right-orders are preserved this gives an upward planar drawing.

It is easy to express "the head of edge $v \rightarrow w$ must be above the tail" as constraints in the IP for bar-visibility representations defined in 4 . Therefore:

Corollary 4. There exists an integer program with $O\left(n^{3}\right)$ variables and constraints to test whether a planar graph has an upward planar drawing. Moreover, the same integer program also finds the minimum-height upward planar drawing.

\section{Conclusion}

This paper considered transformations of one type of planar drawings into another without increasing the height. In particular planar straight-line drawings are equivalent with respect to the height to flat visibility representations or $y$-monotone poly-line drawing, while they are more powerful than either orthogonal drawings or poly-line drawings. The latter two drawing styles are again equivalent with respect to drawing height.

The main gap left open concerns visibility representations. Is it possible to transform any visibility representation into a flat visibility representation of the same height?

As for future problems, it would be interesting to study other drawing objectives, and whether they can be preserved while changing the layout style. Hoffman et al. 11] recently gave some worst-case ratios, but for other graph drawing styles. Is it possible to transform any $y$-monotone poly-line drawing into a straight-line drawing of the same area? The example in Thm. 2 makes this unlikely, but can it be transformed into a straight-line drawing of asymptotically the same area? 


\section{References}

1. Babu, J., Basavaraju, M., Chandran Leela, S., Rajendraprasad, D.: 2-connecting outerplanar graphs without blowing up the pathwidth. In: Du, D.-Z., Zhang, G. (eds.) COCOON 2013. LNCS, vol. 7936, pp. 626-637. Springer, Heidelberg (2013)

2. Biedl, T.: A 4-approximation for the height of drawing 2-connected outer-planar graphs. In: Erlebach, T., Persiano, G. (eds.) WAOA 2012. LNCS, vol. 7846, pp. 272-285. Springer, Heidelberg (2013)

3. Biedl, T., Lubiw, A., Petrick, M., Spriggs, M.J.: Morphing orthogonal planar graph drawings. ACM Transactions on Algorithms 9(4), 29 (2013)

4. Biedl, T., Bläsius, T., Niedermann, B., Nöllenburg, M., Prutkin, R., Rutter, I.: Using ILP/SAT to Determine Pathwidth, Visibility Representations, and other Grid-Based Graph Drawings. In: Wismath, S., Wolff, A. (eds.) GD 2013. LNCS, vol. 8242, pp. 460-471. Springer, Heidelberg (2013)

5. Chimani, M., Zeranski, R.: Upward planarity testing via SAT. In: Didimo, W., Patrignani, M. (eds.) GD 2012. LNCS, vol. 7704, pp. 248-259. Springer, Heidelberg (2013)

6. Dujmovic, V., Fellows, M., Kitching, M., Liotta, G., McCartin, C., Nishimura, N., Ragde, P., Rosamond, F., Whitesides, S., Wood, D.: On the parameterized complexity of layered graph drawing. Algorithmica 52, 267-292 (2008)

7. Felsner, S., Liotta, G., Wismath, S.: Straight-line drawings on restricted integer grids in two and three dimensions. Journal of Graph Algorithms and Applications 7(4), 335-362 (2003)

8. de Fraysseix, H., Pach, J., Pollack, R.: How to draw a planar graph on a grid. Combinatorica 10, 41-51 (1990)

9. Garg, A., Tamassia, R.: On the computational complexity of upward and rectilinear planarity testing. SIAM J. Comput. 31(2), 601-625 (2001)

10. He, X., Wang, J., Zhang, H.: Compact visibility representation of 4-connected plane graphs. Theor. Comput. Sci. 447, 62-73 (2012)

11. Hoffmann, M., van Kreveld, M., Kusters, V., Rote, G.: Quality ratios of measures for graph drawing styles. In: Canadian Conference on Computational Geometry, CCCG 2014 (to appear, 2014)

12. Kant, G.: Drawing planar graphs using the canonical ordering. Algorithmica 16, 4-32 (1996)

13. Lengauer, T.: Combinatorial Algorithms for Integrated Circuit Layout. Teubner/Wiley \& Sons, Stuttgart/Chicester (1990)

14. Miura, K., Nakano, S., Nishizeki, T.: Convex grid drawings of four-connected plane graphs. Int. J. Found. Comput. Sci. 17(5), 1031-1060 (2006)

15. Pach, J., Tóth, G.: Monotone drawings of planar graphs. Journal of Graph Theory 46(1), 39-47 (2004)

16. Rosenstiehl, P., Tarjan, R.E.: Rectilinear planar layouts and bipolar orientation of planar graphs. Discrete Computational Geometry 1, 343-353 (1986)

17. Schnyder, W.: Embedding planar graphs on the grid. In: ACM-SIAM Symposium on Discrete Algorithms (SODA 1990), pp. 138-148 (1990)

18. Biedl, T., Kant, G.: A better heuristic for orthogonal graph drawings. Computational Geometry: Theory and Applications 9, 159-180 (1998)

19. Biedl, T., Kaufmann, M., Mutzel, P.: Drawing planar partitions II: HH-drawings. In: Hromkovič, J., Sýkora, O. (eds.) WG 1998. LNCS, vol. 1517, pp. 124-136. Springer, Heidelberg (1998)

20. Tamassia, R., Tollis, I.: A unified approach to visibility representations of planar graphs. Discrete Computational Geometry 1, 321-341 (1986)

21. Wismath, S.: Characterizing bar line-of-sight graphs. In: ACM Symposium on Computational Geometry (SoCG 1985), pp. 147-152 (1985) 